\title{
The Temperature Dependence of Nitroxide Spin-Label Interaction Parameters: a High-Field EPR Study of Intramolecular Motional Contributions
}

\author{
Anton Savitsky • Matin Plato • Klaus Möbius
}

Received: 30 April 2009/Revised: 2 July 2009/Published online: 14 November 2009

(C) The Author(s) 2009. This article is published with open access at Springerlink.com

\begin{abstract}
High-field W-band electron paramagnetic resonance (EPR) spectroscopy was utilized to study the temperature dependence of the magnetic interaction parameters ( $g$-, hyperfine-, quadrupole tensors) of two types of doublet-state nitroxide spin probes in glass-forming ortho-terphenyl solution: a five-membered ring system of pyrroline type (model for the commonly used methane thiosulfonate spin label) and a six-membered ring system of piperidine type (model for the commonly used TOAC spin label). The analysis of the $g$ - and hyperfine tensors in terms of their isotropic and anisotropic parts reveals at least two mechanisms of motion that are responsible for the temperature dependence of the interaction parameters. The first mechanism is attributed to the overall small-angle motion of the nitroxide molecule in the glassy matrix; it leads to an averaging of the anisotropies of the EPR parameters. The second mechanism originates in an intramolecular out-of-plane motion of oxygen in the nitroxide group. This type of motion is evidenced by comparing the experimental findings for the spin-interaction parameters with the results of density functional theory calculations. The harmonic oxygen out-of-plane vibrations result in a variation of both the isotropic and anisotropic parts of the $g$ - and hyperfine tensors. In contrast, the quadrupole tensor is not influenced by this vibration mechanism in the temperature range under study (90-240 K). Consequences of the applicability of such typical nitroxide radicals for probing details of their protein environment and for studying librational dynamics in frozen solutions are discussed.
\end{abstract}

\footnotetext{
A. Savitsky · K. Möbius $(\square)$

Max-Planck Institute for Bioinorganic Chemistry, 45470 Mülheim, Ruhr, Germany

e-mail: moebius@physik.fu-berlin.de
}

M. Plato · K. Möbius

Department of Physics, Free University Berlin, Arnimallee 14, 14195 Berlin, Germany 


\section{Introduction}

Nitroxide radicals are widely employed in chemical, biochemical, biophysical and biomedical EPR (electron paramagnetic resonance) studies because of the chemical stability of the unpaired electron in the nitroxyl group. A prominent field of application of nitroxide radicals is their use as spin labels attached to otherwise nonparamagnetic molecules to make them susceptible to EPR spectroscopy. Such EPR studies are primarily directed towards detailed information concerning (1) the spatial and electronic structures of the spin-labeled molecule dissolved in suitable matrices such as organic solvents, proteins or membranes, and (2) the various modes of molecular motions of the nitroxide-labeled molecule. The physical and chemical properties of the molecular guest-host system affect the static and dynamic characteristics of the nitroxide spin label. In other words, characteristics of the microenvironment of the studied molecules, which often controls their chemical and biological function, can be probed by EPR on the attached spin label. The information is obtained via an analysis of the measured magnetic interaction parameters ( $g$-, hyperfine- and quadrupole tensors) of the nitroxide reporter group. It is chemically linked to the guest molecules that are dissolved in low concentration in single-crystal hosts, polycrytalline matrices, fluid or frozen solutions with characteristic glass-transition properties.

In the last decades, the development of the site-directed spin labeling (SDSL) mutagenesis technique in conjunction with EPR spectroscopy, as pioneered by Hubbell [1] for standard X-band $(9.5 \mathrm{GHz})$ EPR, has opened a wide field of applications, most prominently in molecular biology. Often spectacular results on structure and dynamics were obtained that would not be attainable by other types of spectroscopy [2-7]. The general approach of the SDSL methodology [1] is to biosynthetically incorporate single cysteine residues into the protein under study to provide reactive sites for the subsequent chemical attachment of nitroxide spin labels that are sulfhydryl-selective. The cysteine sulfhydryl group reacts with the sulfhydryl-functionalized nitroxide reagent, typically the (2,2,5,5,-tetramethyl-1oxypyrrolinyl-3-methyl) methane thiosulfonate (MTS) spin label, to generate a disulfide-linked nitroxide side chain.

Protein function requires, besides proper spatial and electronic static properties of the reaction partners, conformational flexibility ("conformational disorder") resulting from fluctuating intra and intermolecular interactions that are modulated by random motions of "reactant" and "solvent" molecules. Such motions are characterized by their amplitudes and frequencies in their respective energetic substates of slightly different structure. Transitions between conformational substates of the "energy landscape" are thermally driven by the "heat bath" and optimized, via evolutionary adaptation, at physiological temperatures [8-11].

It has been demonstrated by X-ray diffraction, dynamic neutron scattering, and various spectroscopies (optical absorption, nuclear magnetic resonance [NMR], etc.) that proteins undergo a dramatic change in their dynamical and/or structural properties at about $200 \mathrm{~K}[10,12]$. In many respects this transition resembles the behavior of the glass transition of simple molecular glass formers [13, 14]. Well below this glass-transition temperature, $T_{\mathrm{g}}$, proteins are trapped in conformational 
substates, and the internal motions are essentially harmonic. Above $T_{\mathrm{g}}$, however, anharmonic dynamics contribute and, at physiological temperatures, can even dominate the internal fluctuations. Correlations have been found to exist between specific protein functions (such as ligand binding, electron transfer, and proton pumping) and the presence of equilibrium anharmonic motion [15-17]. The application of nitroxide radicals in combination with SDSL methodology can help to understand correlations between protein dynamics and physiological activity at a given temperature.

An important magnetic property of nitroxide radicals that can be measured by EPR is the pronounced anisotropy of the magnetic interaction parameters. They characterize (1) the electron-nuclear hyperfine interaction ( $A$-tensor) of the electron spin with the nitrogen nuclear spin and (2) the combined effect of the electron Zeeman, orbital Zeeman and spin-orbit interactions ( $g$-tensor). Not unexpectedly, the EPR spectra of nitroxides have proven to be very sensitive not only to the static interaction parameters but also to molecular motion in a variety of host matrices. This may lead to partial or even complete averaging of the magnetic interaction anisotropies [18].

However, in order to reliably use the nitroxide label as a sensitive probe for studying complex molecular motion phenomena, one should to be aware of possible contributions from a variety of motional mechanisms that may be active in a particular guest-host system at a particular temperature. Prototypes of such molecular mechanisms are, for example, overall molecular tumbling, spin-label motion around its tether by which it is bonded to the molecule, internal motion of the nitroxide skeleton. All of these motions have specific correlation times and can principally lead to a characteristic temperature dependence of the magnetic interaction parameters of the spin probes. Their EPR responses encode this dynamic information, and sophisticated spectral analysis methods have been developed and described in the literature to disentangle the various modes of motion [19-24].

Temperature-dependent internal nitroxide motion and its effects on EPR spectra were previously observed both in the liquid and solid states, but are revisited in the present study by exploiting the enhanced capabilities of high-field/high-frequency EPR for determining molecular structure and dynamics [5, 25]. We investigated the temperature dependence of the magnetic interaction parameters of the fivemembered ring system of a pyrroline-type nitroxide radical R1 (the head group of the MTS spin label) and the six-membered ring system of a piperidine-type nitroxide R2 (OH-TEMPO) as a model of the non-natural amino-acid TOAC spin label (2,2,6,6-tetramethylpiperidine-1-oxyl-4-amino-4-carboxylic acid, see Fig. 1). The two nitroxides were dissolved in $\mathrm{mM}$ concentration in glass-forming orthoterphenyl solution and studied in the temperature range of $90-240 \mathrm{~K}$ by $\mathrm{W}$-band (95 GHz) high-field EPR. In these experiments, the canonical $g$-tensor components in the frozen-solution nitroxide spectrum become fully resolved, and the principal components of the $g$ - and $A$-tensors can be determined with high accuracy. Thereby, it becomes possible to differentiate between overall motion of the nitroxide molecule itself and intramolecular motion of the nitroxide skeleton. Ortho-terphenyl as the host matrix was chosen for two reasons. First, it has a relatively high transition temperature $\left(T_{\mathrm{g}}=243 \mathrm{~K}\right)$ to the glass phase, which allows to study the 
system over a wide temperature range where only harmonic motion is allowed. Second, ortho-terphenyl is strictly apolar and aprotic, i.e., it neither carries an electric dipole moment nor has hydrogen-bonding capability. This property nicely prevents complications in EPR data analysis owing to possible contributions from temperature-dependent variations of the nitroxide's matrix environment in terms of polarity and proticity $[5,25]$.

\section{Experimental}

\subsection{Materials}

The molecular structure of the nitroxide spin labels R1 (3-hydroxymethyl-2,2,5,5tetramethylpyrrolin-1-oxyl) and R2 (4-hydroxy-2,2,6,6-tetramethylpiperidine-Noxyl) are shown in Fig. 1. The R1 radical in the protonated and perdeuterated R1- $\mathrm{D}_{15}(\mathrm{OH})$ form was synthesized as described previously [26]. Both forms of R1 were kindly provided by Herbert Zimmermann (Max-Planck Institute for Medical Research, Heidelberg). The protonated nitroxide R2 (Aldrich, 97\%) and perdeuterated $\mathrm{R} 2-\mathrm{D}_{17}(\mathrm{OH})$ (Aldrich, 97\% D, 95\%) were used as delivered. Benzene- $\mathrm{D}_{6}$ was obtained from Fluka and Aldrich in its purest commercially available form and used without further purification. Ortho-terphenyl-D ${ }_{14}(98 \%$ D) was supplied by Cambridge Isotope Laboratories.

The nitroxide spin label (R1 or R2) was dissolved in benzene to obtain a stock solution for sample preparations. This stock solution was mixed with powdered orthoterphenyl, and the benzene solvent was then evaporated under air. The resulting mixed powder, containing $1 \mathrm{mM}$ of nitroxide radical, was heated to about $65^{\circ} \mathrm{C}$ to become fluid and then transferred into the sample quartz capillary (inner diameter, $0.6 \mathrm{~mm}$ ). A glass-type frozen solution was obtained by shock freezing the sample with liquid nitrogen. The cold sample was finally transferred into the precooled W-band EPR cavity. The temperature was increased in 10-20 K steps starting from $90 \mathrm{~K}$.

\subsection{EPR Methods}

The EPR measurements were performed with a laboratory-built W-band spectrometer operating in both continuous-wave (cw) and pulsed modes at an
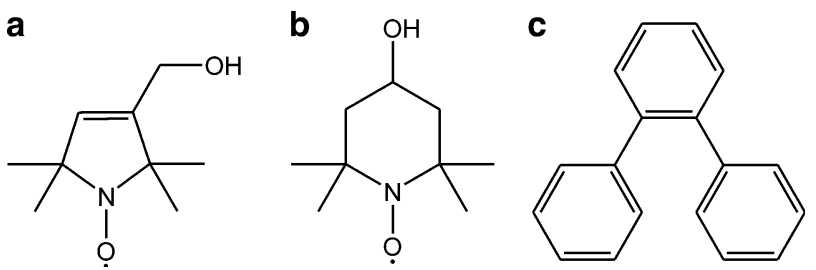

Fig. 1 Molecular structure of the studied nitroxide radical of pyrroline type R1 (a) and of piperidine type R2 (b). c Ortho-terphenyl host for diluted nitroxide solutions 
EPR frequency of $95 \mathrm{GHz}$ and an external magnetic field of about $3.4 \mathrm{~T}[5,27]$. For cw EPR experiments, a gold plated bronze $\mathrm{TE}_{011}$ cavity was used (loaded quality factor $Q_{\mathrm{L}}=2400$ of the empty cavity, temperature independent in the range of $90-290 \mathrm{~K}$ ). The external $B_{0}$ field was modulated at $8 \mathrm{kHz}$ with an amplitude of $30 \mu \mathrm{T}$.

In order to avoid cw EPR line shape distortions due to saturation and rapidpassage effects, the absorption and dispersion EPR responses in both the $0^{\circ}$ and $90^{\circ}$ phases relative to the modulation phase were acquired simultaneously. The microwave $(\mathrm{mw})$ power incident on the critically coupled loaded cavity was optimized by minimizing the integral signal component in both dispersion channels to minimize rapid-passage effects [28-30]. The $0^{\circ}$-dispersion signal was checked to reproduce a true absorption signal after Hilbert transformation to exclude powersaturation effects [31].

The pulsed EPR experiments were done using a high- $Q$ mw cavity manufactured from $99.9 \%$ gold. The two-pulse echo decays were acquired using the Hahn-echo sequence $\left(t_{\mathrm{p}}\right)-\tau-\left(2 t_{\mathrm{p}}\right)$-echo. The phase of the first mw pulse was cycled between $[+x ;-x]$ as it is common practice to avoid signal distortions [32]. The decay amplitudes were obtained by integrating the echo responses over a time window, centered at the echo maximum that covers $60 \%$ of the echo intensity to optimize resolution and signal-to-noise ratio. The interpulse separation time $\tau$ was varied in 50 steps from $100 \mathrm{~ns}$ to full echo decay.

In electron spin echo envelope modulation (ESEEM) measurements, the stimulated echo mw pulse sequence $\left(t_{\mathrm{p}}\right)-\tau-\left(t_{\mathrm{p}}\right)-T-\left(t_{\mathrm{p}}\right)$-echo with a repetition rate of $1 \mathrm{kHz}$ was used. The pulse length for a $\pi / 2$ pulse, $t_{\mathrm{p}}$, and the separation between the first and the second pulses in the sequence, $\tau$, were generally set to 25 and $40 \mathrm{~ns}$, respectively. The time interval $T$ was incremented in $5 \mathrm{~ns}$ steps starting with $T_{0}=30 \mathrm{~ns}$. The single echo response was measured with the detector bandwidth limited to $120 \mathrm{MHz}$, and the commonly used four-step phase cycling $[+x ;-x]$ of the first and second mw pulses was employed to avoid unwanted primary echos and free induction decay distortions [32]. The full echo response was stored in the computer for later evaluation. The ESEEM data sets were obtained subsequently by stepping the external magnetic field through the nitroxide spectrum $(0.2 \mathrm{mT}$ per step). They were evaluated as described previously [26].

\subsection{Spectra Analysis and Quantum Chemical Calculations}

All experimental spectra analysis and simulation procedures were performed on the basis of the EasySpin toolbox for the Matlab program package [33, 34]. Quantum chemical calculations of magnetic interaction parameters such as the hyperfine $(A)$ and quadrupole $(Q)$ tensors (which represent local probes of the electronic structure) and the $g$-tensor (which represents a global probe of the electronic structure) of the nitroxide radicals were performed on the density functional theory (DFT) level with the quantum-chemical program package ORCA developed by F. Neese $[35,36]$. Detailed information on the general features of ORCA is available through www.thch.uni-bonn.de/tc/orca. 


\section{Results}

\subsection{EPR Measurements}

Figure 2 shows W-band cw EPR spectra of R1-D $\mathrm{D}_{15}$ and $\mathrm{R} 2-\mathrm{D}_{17}$ nitroxide radicals measured at different temperatures in frozen ortho-terphenyl- $\mathrm{D}_{14}$ solution. The spectra exhibit the typical powder-pattern line shape expected for a dilute distribution of nitroxides at high magnetic fields.

The spectra are clearly resolved into three separate regions corresponding to the principal values of the $g$-tensor, i.e., $g_{x x}, g_{y y}$ and $g_{z z}$. Moreover, due to the narrowing of the inhomogeneous line width by perdeuteration of the radicals and host molecules, the nitrogen ${ }^{14} \mathrm{~N}$ hyperfine splitting (triplets, $I=1$ ) is clearly observed in all $g$-regions. Comparison of the changes in the EPR spectra upon temperature variation reveals similarity and differences for both radicals. The behavior in the $g_{z z}$-region (high-field region) is similar in both systems. At higher temperatures, the central EPR line $\left(M_{I}=0\right)$ shifts towards the center of the spectrum, indicating an increased $g_{z z}$-value. Also the line splitting within the hyperfine triplet slightly decreases, i.e., $A_{z z}$ decreases. The major differences are observed in the low-field $g_{x x}$-region. The $A_{x x}$ triplet of the R2 radical (see Fig. $2 \mathrm{~b}$ ) does not reveal any changes in line shape and shifts to higher fields upon temperature increase, i.e., the $g_{x x}$-value decreases. In contrast, for the R1 radical, the $g_{x x}$-value does not change, but the distorted line shape of the $A_{x x}$ triplet indicates an increase of the $A_{x x}$ value at higher temperature (see Fig. 2a).

The exact evaluation of the $g$ - and $A$-tensor values from the cw EPR spectra requires a multi-parameter fit of the theoretical spectra, as obtained from a numerical solution of the spin Hamiltonian, containing the nuclear Zeeman [37] and ${ }^{14} \mathrm{~N}$ quadrupole [26] interaction terms, to the experimental spectra. Fortunately, the

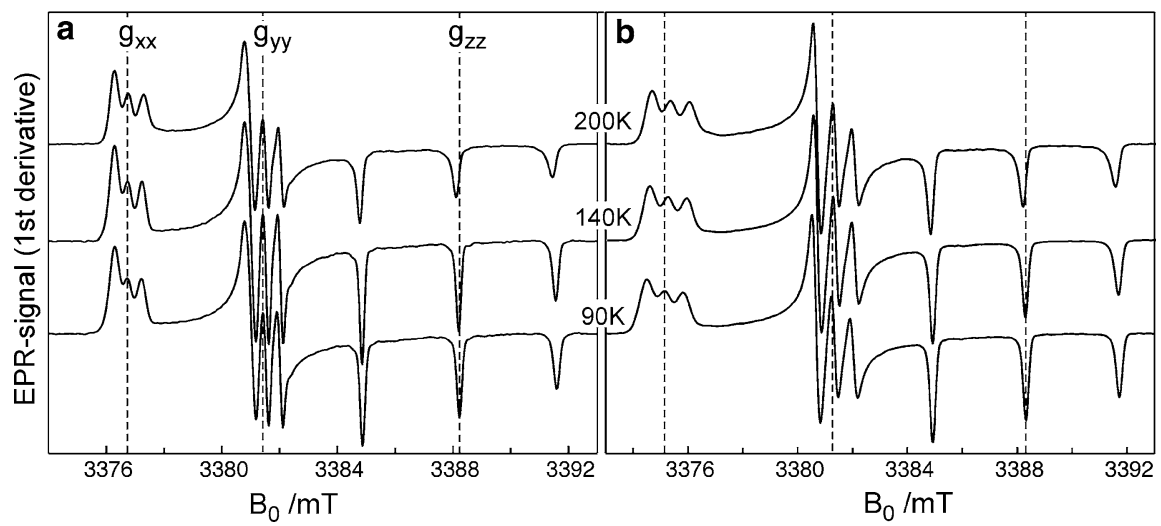

Fig. 2 Experimental W-band cw EPR spectra of $1 \mathrm{mM}$ R1-D $\mathrm{D}_{15}$ (a) and R2-D 17 (b) nitroxide radicals in frozen solution of ortho-terphenyl- $\mathrm{D}_{14}$ taken at 90,140 , and $200 \mathrm{~K}$. The field positions corresponding to $g_{x x^{-}}, g_{y y^{-}}$and $g_{z z^{-}}$values at $90 \mathrm{~K}$ are marked by the dashed lines 
high spectral resolution at $\mathrm{W}$-band allows to analyze independently the spectra in the regions of the canonical $g$-values. Moreover, precise information on $A_{x x}$ and $A_{y y}$ as well as on the ${ }^{14} \mathrm{~N}$ quadrupole interaction tensor can be obtained from an analysis of 3-pulse ESEEM spectra, as it was shown previously [26].

The ESEEM spectra of the R1 and R2 radicals at 90 and $180 \mathrm{~K}$ are shown in Fig. 3. This figure displays the frequency-field dependence of the 3-pulse stimulated echo ESEEM of R2-H $\mathrm{H}_{18}$ (Fig. 3a) and R1-D ${ }_{15}$ (Fig. 3b) in ortho-terphenyl-D ${ }_{14}$ in the $g_{x x}-\mathrm{g}_{y y}$ spectral region. The echo decay functions $S\left(2 \tau, B_{0}\right)$ were recorded at 50 field positions with $0.2 \mathrm{mT}$ separation. The protonated form of R2 was used to avoid the strong ${ }^{2} \mathrm{H}$ ESEEM line which, at W-band, appears at $22.1 \mathrm{MHz}$. The spectra of the $\mathrm{R} 2$ radical are found to be identical at both temperatures (see Fig. 3a). This shows that the parameters of neither the hyperfine interaction $\left(A_{x x}=19.45 \pm 0.15\right.$ $\left.\mathrm{MHz}, \quad A_{y y}=18.9 \pm 0.15 \mathrm{MHz}\right)$ nor the quadrupole interaction $\left(Q_{x x}=\right.$ $+1.22 \pm 0.03 \mathrm{MHz}, Q_{y y}=+0.54 \pm 0.03 \mathrm{MHz}$ ) are temperature dependent within experimental error. For the $\mathrm{R} 1$ radical, the quadrupole-tensor components $\left(Q_{x x}=+1.26 \mathrm{MHz}, Q_{y y}=+0.53 \mathrm{MHz}\right)$ also do not reveal any temperature dependence, in agreement with a previous study [26]. The hyperfine-tensor
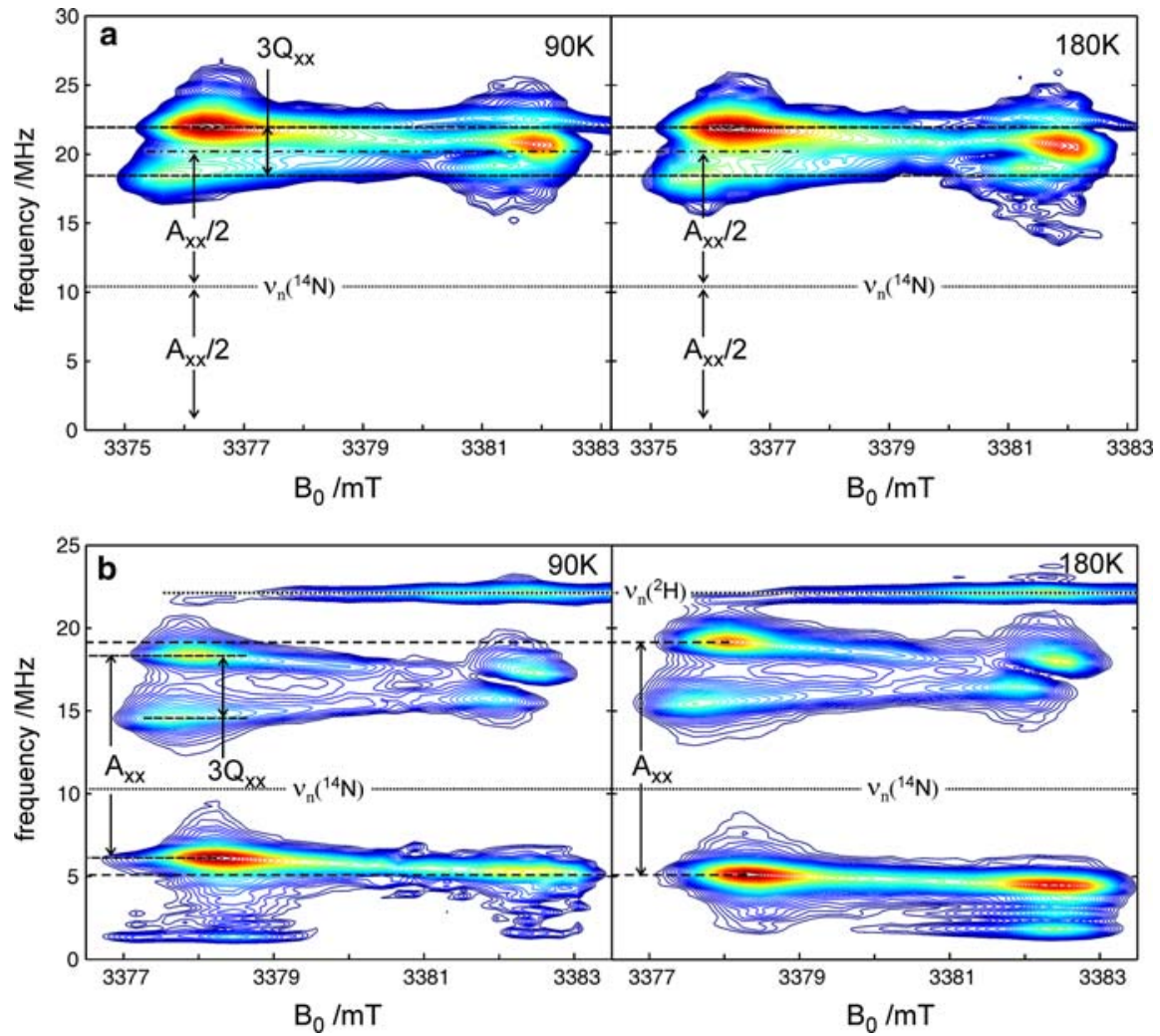

Fig. 3 Frequency-field dependence of the 3-pulse stimulated echo ESEEM of R2-H $\mathrm{H}_{18}$ (a) and R1-D ${ }_{15}$ (b) in ortho-terphenyl-D $\mathrm{D}_{14}$ at 90 and $180 \mathrm{~K}$ in the $g_{x x}-g_{y y}$ spectral region. The contour lines are equidistant from 0.05 to 1 times the maximum Fourier-transformed ESEEM intensity 
components, however, increase markedly when going from $90 \mathrm{~K}\left(A_{x x}=12.1 \mathrm{MHz}\right.$, $\left.A_{y y}=12.2 \mathrm{MHz}\right)$ to $180 \mathrm{~K}\left(A_{x x}=13.8 \mathrm{MHz}, A_{y y}=13.5 \mathrm{MHz}\right)$. This is in agreement with the cw EPR observations.

Using the observables from ESEEM, a detailed analysis of the cw EPR spectra in terms of the numerical solution of the spin Hamiltonian was performed. Satisfactory fits of calculated spectra to experimental ones were obtained for R1 up to $220 \mathrm{~K}$, and for R2 up to $240 \mathrm{~K}$. At higher temperatures, the cw EPR spectra become distorted and cannot be described in terms of averaged powder spectra. Previous studies of the guest-molecule dynamics in glass-forming solvents, in particular ortho-terphenyl [38], showed that rotational motion of the guest molecule continues even below the glass-transition temperature of the host. Therefore, in order to determine the temperature region in which motional effects become the dominant factor in affecting the cw EPR line shape, the 2-pulse echo decay surfaces were examined at different temperatures. The spin-echo experiments are a more sensitive monitor of motional processes than $\mathrm{cw}$ EPR. The echo experiment probes directly the homogeneous line width at each spectral position affected by the motional process [39-41]. The cw experiment, on the other hand, detects the static inhomogeneous line width dominated by unresolved hyperfine couplings and, therefore, is less sensitive to motional contributions.

Figure $4 \mathrm{a}$ shows the field dependence of the inverse phase memory time, $1 / T_{\mathrm{m}}$, of nitroxide $\mathrm{R} 2$ at different temperatures. $T_{\mathrm{m}}$ values were extracted from individual echo decays by a monoexponential fit procedure according to

$$
S\left(2 \tau, B_{0}\right)=S\left(0, B_{0}\right) \cdot \exp \left[-\frac{2 \tau}{T_{\mathrm{m}}\left(\mathrm{B}_{0}\right)}\right]
$$

At temperatures below $220 \mathrm{~K}, T_{\mathrm{m}}$ shows a weak field dependence. The echo decay appears to be faster in the maximum of the EPR spectral density. Such a
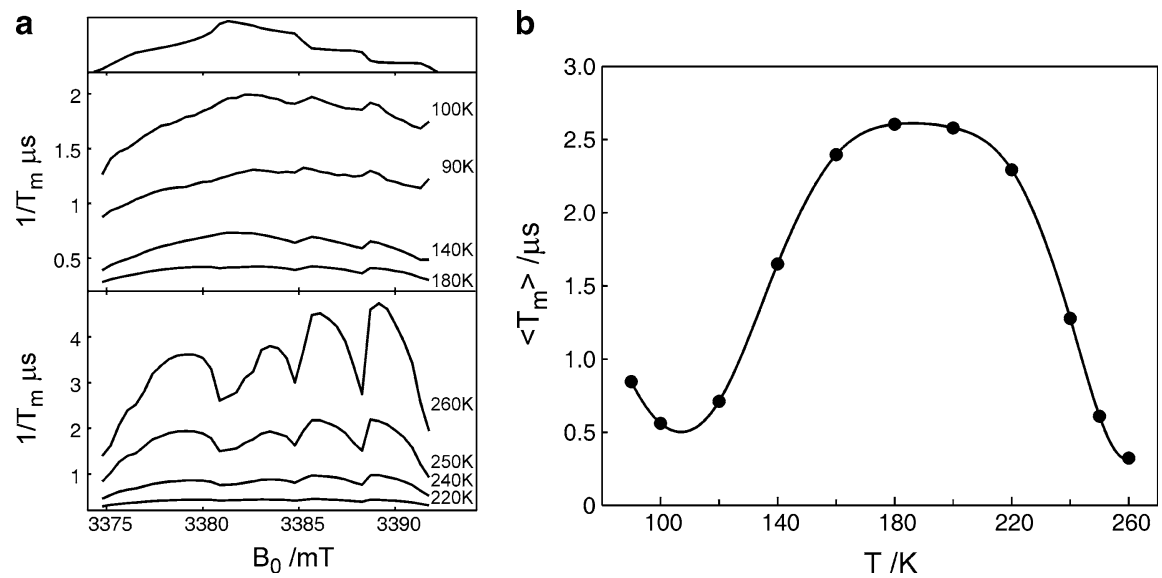

Fig. 4 a Inversed phase memory time $1 / T_{\mathrm{m}}$ as a function of magnetic field obtained at different temperatures for the $\mathrm{R} 2-\mathrm{D}_{17}$ radical. The echo-detected EPR spectrum of $\mathrm{R} 2$ is shown on the top as reference to the spectral positions. b Temperature dependence of the average spin-echo phase memory relaxation time $\left(<T_{\mathrm{m}}>\right)$ as extracted from exponential Hahn echo decay profiles and averaged over different positions in the W-band EPR spectrum of R2- $\mathrm{D}_{17}$ in ortho-terphenyl- $\mathrm{D}_{14}$ 
behavior is typical in cases when the anisotropy of $T_{\mathrm{m}}$ is determined by spectral diffusion processes, for instance, instantaneous diffusion, diffusion due to nuclear spin flips, etc. [42]. The field dependence of $T_{\mathrm{m}}$ changes at elevated temperatures above $220 \mathrm{~K}$. The phase memory time exhibits pronounced maxima in field positions corresponding to orientations with the principal axes of $g_{x x(y y, z z)}$ parallel to $B_{0}$. This is likely to be caused by dynamical molecular processes occurring within the mw pulse separation time. The field positions, corresponding to orientations where the external magnetic field is not collinear with a principal axis of the $g$-tensor, are more affected by the angular fluctuations of the radical than those corresponding to the canonical orientations. This is because the stochastic smallangle motion of the molecule causes fluctuations of the Larmor frequency of the observed spins which are proportional to changes of the orientation-dependent $g$-value, and these changes are smallest for the canonical orientations. The larger shifts of the Larmor frequency at intermediate orientations accelerate the dephasing of the echo during the pulse separation time. Thus, the behavior of the phase memory time at higher temperatures can be described in terms of the small-angle reorientation (libration) motion of the radical. Additionally, the measured $T_{\mathrm{m}}$ field dependence shows that such a librational motion does not occur along any preferential principal axis, because the $T_{\mathrm{m}}$ values at any of the $g_{i i}$ positions are practically equal.

This analysis of the phase memory time anisotropy is consistent with the behavior of its isotropic part, which can be approximated by $<T_{\mathrm{m}}>$. Figure $4 \mathrm{~b}$ shows the temperature dependence of these averaged $<T_{\mathrm{m}}>$ values. Below $140 \mathrm{~K}$, $<T_{\mathrm{m}}>$ decreases because the rate of rotation of the methyl groups attached to the nitroxide ring becomes comparable with the electron-nuclear hyperfine coupling of the inequivalent methyl deuterons [43]. Above $220 \mathrm{~K},<T_{\mathrm{m}}>$ increases due to the increase in the rate of molecular motion of the nitroxide.

Thus, the molecular motion is expected not to influence significantly the cw EPR spectra of the R2 radical up to about $240 \mathrm{~K}$, which is consistent with the results of fitting the cw EPR spectra. The analysis of the echo decays from the R1 radical shows that intramolecular motion becomes pronounced at slightly lower temperatures. However, the overall relaxation picture is in good agreement with the R2 case. Therefore, in the following considerations the results for the $\mathrm{R} 1$ radical up to $220 \mathrm{~K}$ are used.

From the combined analysis of the cw EPR and ESEEM spectra, the $g$ - and $A$-tensors for the $\mathrm{R} 1$ and $\mathrm{R} 2$ radicals at different temperatures were evaluated with high accuracy. In principle, the temperature dependence of the magnetic interaction parameters of the nitroxides can be caused by several mechanisms. (1) The overall motion of the nitroxide (either tumbling or librations) will lead to an averaging of the anisotropies and will not influence the isotropic magnetic parameters [18]. (2) The intramolecular motion will generally influence both anisotropic and isotropic parameter values. (3) The temperature dependence of the static environment (changing, for example, the H-bond network and/or the polarity) also influences both parts. The role of the last mechanism, however, is likely to be negligible in ortho-terphenyl due to the absence of any H-bond forming fragments and due to its nonpolarity. Thus, to differentiate between overall (1) and intramolecular (2) motion 
of the nitroxides, the $g$ - and $A$-tensors have to be analyzed in terms of their isotropic values

$$
g_{\text {iso }}=\frac{g_{x x}+g_{y y}+g_{z z}}{3} ; \quad A_{\text {iso }}=\frac{A_{x x}+A_{y y}+A_{z z}}{3}
$$

and their anisotropies. The hyperfine anisotropy is determined by the dipolar hyperfine interaction of the electron spin with the ${ }^{14} \mathrm{~N}$ nuclear spin, and is given by

$$
D=\frac{A_{z z}-\left(A_{x x}+A_{y y}\right) / 2}{3} .
$$

We assume axial symmetry of the $A$-tensor; its principal values are then given by

$$
A_{z z}=A_{\text {iso }}+2 D ; \quad A_{x x, y y}=A_{\text {iso }}-D \text {. }
$$

Figure 5 illustrates the temperature dependence of the $g_{\text {iso, }} A_{\text {iso }}$ and $D$ parameters, as evaluated from the experimental data of the $g$ - and $A$-tensors. The $D$ value decreases for both $\mathrm{R} 1$ and $\mathrm{R} 2$ radicals with increasing temperature, as is
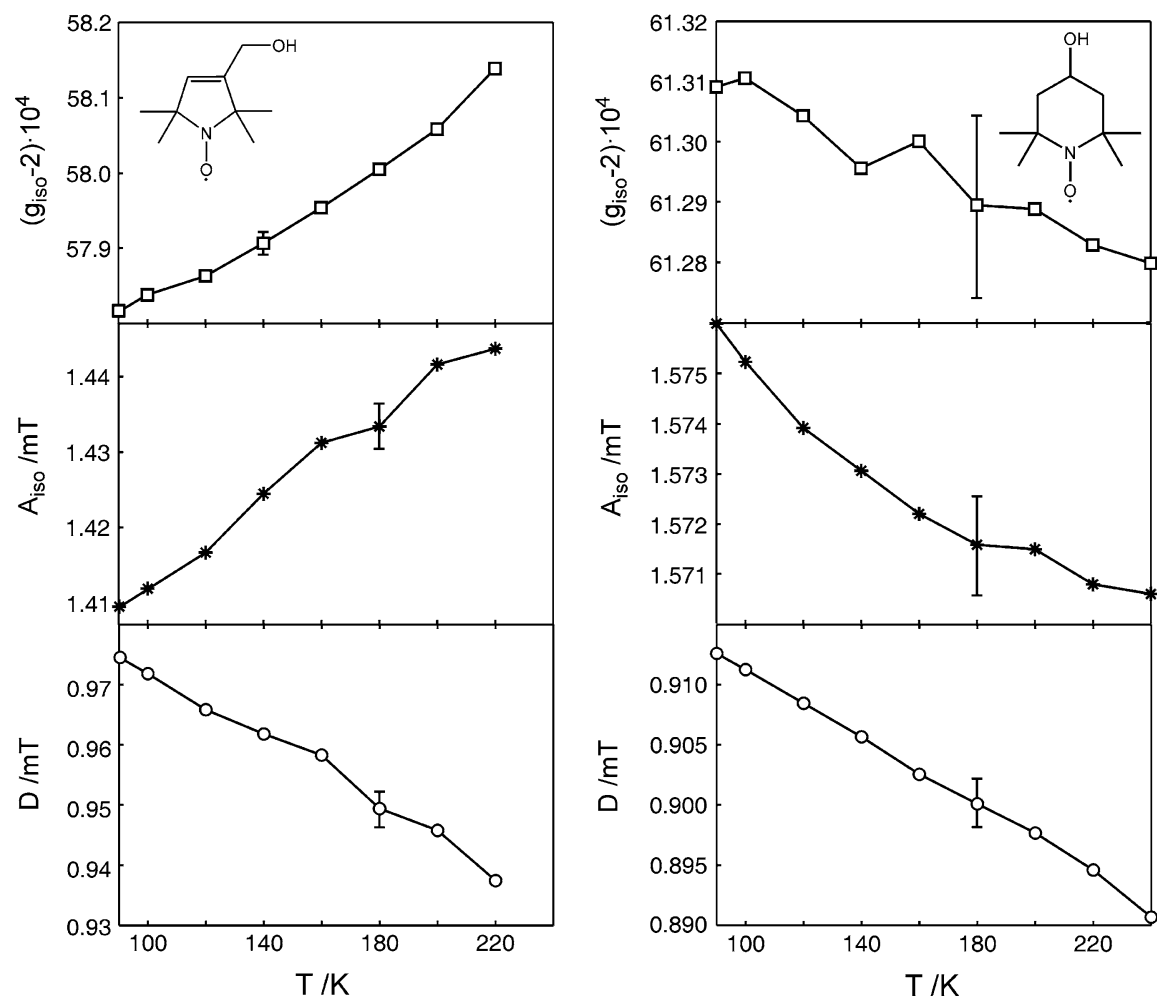

Fig. 5 Temperature dependences of $g_{\text {iso }}$ (open square), $A_{\text {iso }}$ (asterisk) and D (open circle) of the R1 (left) and R2 (right) nitroxide radicals, as obtained from the combined analysis of the experimental W-band cW EPR and ESEEM spectra. For simplicity, the error margins are shown only for the respective parameter values at $180 \mathrm{~K}$ 
expected from the librational model. However, the temperature variations of $g_{\text {iso }}$ and $A_{\text {iso }}$ demonstrate the importance of the intramolecular motion mechanism in both radical systems. Moreover, the experimental results in Fig. 5 show the striking difference of the temperature behavior of $A_{\text {iso }}$ and $g_{\text {iso }}$ in the opposite signs of the temperature gradients, $\mathrm{d} g_{\text {iso }}(T) / \mathrm{d} T$ and $\mathrm{d} g_{\text {iso }}(T) / \mathrm{d} T$, for $\mathrm{R} 1$ and $\mathrm{R} 2: \mathrm{d} g_{\text {iso }}(T) / \mathrm{d} T>0$ and $\mathrm{d} g_{\text {iso }}(T) / \mathrm{d} T>0$ for $\mathrm{R} 1$, whereas $\mathrm{d} A_{\text {iso }}(T) / \mathrm{d} T<0$ and $\mathrm{d} g_{\text {iso }}(T) / \mathrm{d} T<0$ for $\mathrm{R} 2$ (although for R2 the temperature dependence of $g_{\text {iso }}$ is only very weak). The linearly approximated gradients for R1 and R2 are summarized in Table 1.

For the temperature dependence of $g_{\text {iso }}$ of R1 and R2 in organic solvents, there are no data of comparable accuracy in the literature till now. Our results for $\mathrm{d} A_{\text {iso }}(T) / \mathrm{d} T$, however, can be compared with earlier findings from the X-band EPR and electron-nuclear double resonance investigations of a series of pyrroline and piperidine NO radicals in fluid solution [44-47]. Apart from one or two exceptions found in chloroform solutions, all radicals of the pyrroline type, like R1, show positive temperature coefficients, whereas those of the piperidine radicals, like R2, are negative. The difference in the slopes was explained by out-of-plane vibrations of the oxygen atom about nitrogen and differences of the radical geometries [44]. Indeed, the crystallographic studies of pyrroline-type radicals reveal the planar structure of the radical ring, and that the $\mathrm{N}-\mathrm{O}$ bond lies in the $\mathrm{C}-\mathrm{N}-\mathrm{C}$ plane [4851]. In contrast, piperidine-type radicals typically adopt a chair-like conformation of the ring with an $\mathrm{N}-\mathrm{O}$ bond angle with the $\mathrm{C}-\mathrm{N}-\mathrm{C}$ plane, $\alpha$, of about $20^{\circ}$ [49, 52-54]. Thus, taking into account the symmetry of out-of-plane vibrations, the $A_{\text {iso }}$ value averaged over all occupied vibrational states increases with temperature for planar structures due to an increased averaged out-of-plane angle $\left\langle\alpha^{2}\right\rangle$. In contrast, the averaged $A_{\text {iso }}$ value decreases for pyramidal structures due to decreasing $\left\langle\alpha^{2}\right\rangle$ [44]. The DFT calculations of the energy profiles [55], in conjunction with theoretical investigations of the magnetic interaction parameters for different out-of-plane angles [56, 57], suggest the importance of the out-ofplane vibrational mechanism for the temperature dependence of the $A_{\text {iso }}, g_{\text {iso }}$ and $D$ observables.

We note that for pyrroline-type radicals in fluid solution the $\mathrm{d} A_{\text {iso }}(T) / \mathrm{d} T$ values of +0.14 to $+0.20 \mu \mathrm{T} \mathrm{K}^{-1}$, as reported for different aprotic solvents [44, 46, 47], are comparable to that of $+0.28 \mu \mathrm{T} \mathrm{K} \mathrm{K}^{-1}$ in frozen ortho-terphenyl solution (see Table 1). However, for the piperidine-type $\mathrm{R} 2$ radical, slopes -0.18 to

Table 1 Theoretical and experimental temperature coefficients of the magnetic parameters of the nitroxide radicals R1 and R2 in frozen ortho-terphenyl solution: isotropic ${ }^{14} \mathrm{~N}$ hyperfine interaction, $A_{\text {iso, }}$, anisotropic ${ }^{14} \mathrm{~N}$ hyperfine interaction, $D$, and isotropic Zeeman interaction, $g_{\text {iso }}$ (for details see the text)

\begin{tabular}{lllllll}
\hline Parameter & Units & $\mathrm{R} 1$ & & \multicolumn{2}{l}{$\mathrm{R} 2$} & \\
\cline { 5 - 6 } & & Theory & Experiment & & Theory & Experiment \\
\hline$\frac{\mathrm{d} A_{\text {iso }}}{\mathrm{d} T}$ & $\mu \mathrm{T} \mathrm{K}^{-1}$ & +0.5 & $+(0.28 \pm 0.03)$ & & -0.07 & $-(0.05 \pm 0.03)$ \\
$\frac{\mathrm{d} g_{\text {iso }}}{\mathrm{d} T}$ & $10^{-8} \mathrm{~K}^{-1}$ & +30 & $+(24 \pm 3)$ & & +3 & $-(2 \pm 2)$ \\
$\frac{\mathrm{d} D}{\mathrm{~d} T}$ & $\mu \mathrm{T} \mathrm{K}^{-1}$ & -0.07 & $-(0.28 \pm 0.03)$ & & +0.01 & $-(0.14 \pm 0.03)$ \\
\hline
\end{tabular}


$-0.34 \mu \mathrm{T} \mathrm{K}^{-1}$ have been obtained in liquids [44-47]. They are significantly larger than the value of $-0.05 \mu \mathrm{T} \mathrm{K} \mathrm{K}^{-1}$ measured in glassy ortho-trephenyl. However, the comparisons of $\mathrm{d} A_{\mathrm{iso}}(T) / \mathrm{d} T$ in liquids and solids have to be made with caution. On one side, the temperature dependence of radical-solvent interactions in liquid solution [47] as well as changes of the solvent properties, e.g., solvent volume [58], have to be taken into account. On the other hand, additional intramolecular processes, e.g., the fluctuations of the chair structure of the piperidine nitroxide ring, can influence the magnetic parameters in liquids.

In the following, we present the results of DFT calculations on R1 and R2 to establish their energy-minimized structures and to obtain theoretical estimates on the temperature dependence of the observed EPR interaction parameters due to the out-of-plane bending vibration as the dominating intramolecular motional mechanism.

\subsection{DFT Calculations}

In the case of the R1 radical, DFT results obtained by ORCA [35, 36] calculations were already reported earlier [26], including computational details and ORCA program settings used therein. Nevertheless, in the following we shall present a summary of our approach to these calculations, which were performed identically on both R1 and R2. This part includes the more thorough consideration of the out-of-plane bending motion of the $\mathrm{N}-\mathrm{O}$ bond as an important structural feature of these radicals. Moreover, the characteristics of this out-of-plane motion are spectroscopically strongly distinguishing between these two nitroxide radicals.

\subsubsection{Energies}

All hyperfine-, quadrupole- and g-tensor calculations were preceded by an ORCA optimization procedure to establish the minimum-energy molecular structures of R1 and R2. These calculations were performed for the in-vacuo case without imposing any geometrical constraints. The chosen ORCA input settings for the energy calculations were spin unrestricted self-consistent field (SCF, unrestricted KohnSham), DFT hybrid functional B1LYP, Ahlrich's split-valence basis set with polarization (SVP) [59], "Tight SCF convergence" with $\Delta E=5 \times 10^{-6}$ a.u.

The starting geometries were obtained by a Molecular Modeling software (Hyperchem, Professional Version, Release 6, Hypercube Inc.) on a semiempirical level (PM3).

Special geometrical features of $\mathrm{R} 1$ and $\mathrm{R} 2$, evolving from these in-vacuo calculations, are:

R1 Near planarity of the five-membered ring (see Fig. 6) with a maximum twist angle ca. $1^{\circ}$, bond length $r(\mathrm{~N}-\mathrm{O})=1.262 \AA$, negligibly small bending angle $\alpha$ of the NO bond with respect to the ring plane

$\mathrm{R} 2$ Chair conformation of the six-membered ring with bond length $r(\mathrm{~N}-\mathrm{O})=$ $1.272 \AA$, out-of-plane bending angle $\alpha=-19^{\circ}$ with respect to the adjacent 


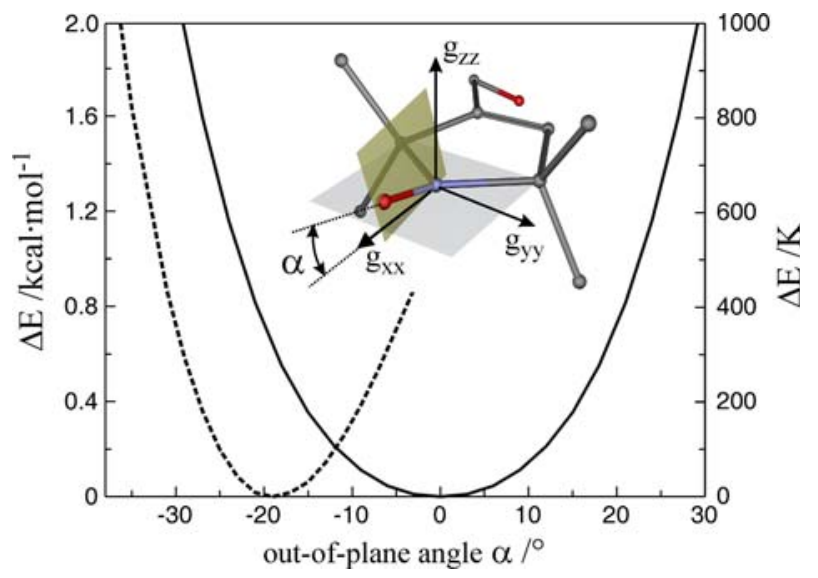

Fig. 6 Relaxed energy profile as a function of the out-of-plane angle $\alpha$ calculated for the R1 (solid line) and R2 (dashed line) nitroxide radicals. Inset shows the structure of R1 and visualizes the definition of the out-of-plane angle $\alpha$

$\mathrm{C}-\mathrm{N}-\mathrm{C}$ plane, the negative sign meaning that the bent is inward as seen from the side of the chair ("equatorial" $\mathrm{N}-\mathrm{O}$ bond)

In addition to the energy-minimized structures of R1 and R2, we also calculated the minimum "relaxed" potential-energy profiles $E(\alpha)$ by retaining the original ORCA program settings but constraining the out-of-plane bending angle $\alpha$ to a set of fixed values around the equilibrium value $\left(0^{\circ}\right.$ and $-19^{\circ}$ for $\mathrm{R} 1$ and $\mathrm{R} 2$, respectively).

The result of these calculations for the relaxed energy profiles is presented in Fig. 6. They reveal that in both radicals R1 and R2 a large range of angles $\alpha$ of around $\pm 10^{\circ}$ is covered within a low-energy range of ca. $0.5 \mathrm{kcal} / \mathrm{mol}$. This corresponds to $200 \mathrm{~K}$, thus establishing the out-of-plane bending angle $\alpha$ as a comparatively "soft" structural parameter.

Whereas the relaxed potential-energy profile $E(\alpha)$ for R1 is symmetrical around the equilibrium angle $\alpha=0^{\circ}$, as is expected by symmetry, R2 shows an unsymmetrical profile with lower energy values for angles approaching planarity (right branch $\alpha>=-19^{\circ}$ ). This asymmetry is responsible for a sign change in the temperature gradient of $A_{\text {iso }}(T)$ between the two radicals R1 and R2 (see below).

Our calculated energy profiles are essentially consistent with DFT results found for structurally similar nitroxide systems by Komaromi et al. [55]. For the piperidine- $N$-oxyl radical, which, like $\mathrm{R} 2$, has a six-membered ring in chair conformation, these authors obtained an equilibrium angle $\alpha=-26^{\circ}$ (termed "equatorial" $\mathrm{N}-\mathrm{O}$ bond). However, they also obtained a pronounced relative energy minimum at $\alpha \approx+30^{\circ}$ (termed "axial" $\mathrm{N}-\mathrm{O}$ bond) and $\Delta E \approx 0.5 \mathrm{kcal} / \mathrm{mol}$. We were not able to confirm the existence of such an energetically nearby invertomer in the case of R2, even when applying the same DFT functional (BLYP) and basis set $\left(6-31 \mathrm{G}^{*}\right)$ as used by Komaromi et al. [55]. Apparently, in R2 there exists additional 
structural stabilization of the equatorial conformation by the tertiary butyl groups that are attached to the $\mathrm{C}$-atoms neighboring the $\mathrm{N}-\mathrm{O}$ bond.

\subsubsection{EPR Interaction Parameters}

The ORCA input settings for the DFT calculations of the various interaction tensors in the spin Hamiltonian were chosen as follows:

(1) $A$ - and $Q$-tensors: the same as for the energy calculations except for choosing the Kutzelnigg basis set IGLO-III for NMR and EPR calculations [60]; an integration grid ' 5 ' for high numerical accuracy in the nuclear regions.

(2) g-tensor: spin unrestricted SCF; local spin density (LSD) functional; Ahlrichs' split-valence basis set (SVP) [59]; resolution of the identity (RI) approximation; RI-SOMF(1X) treatment of the spin-orbit coupling operator; orbital window selection, -100 to +100 a.u.

The ORCA output produces isotropic and/or anisotropic (traceless) contributions to all three tensors and gives the directions of the principal axes in the chosen molecular axes system.

Figure 7 depicts the calculated values of $A_{\text {iso }}, g_{\text {iso }}$ and of the dipolar hyperfine coupling $D$ as a function of the out-of-plane angle $\alpha$ for both R1 and R2 in their
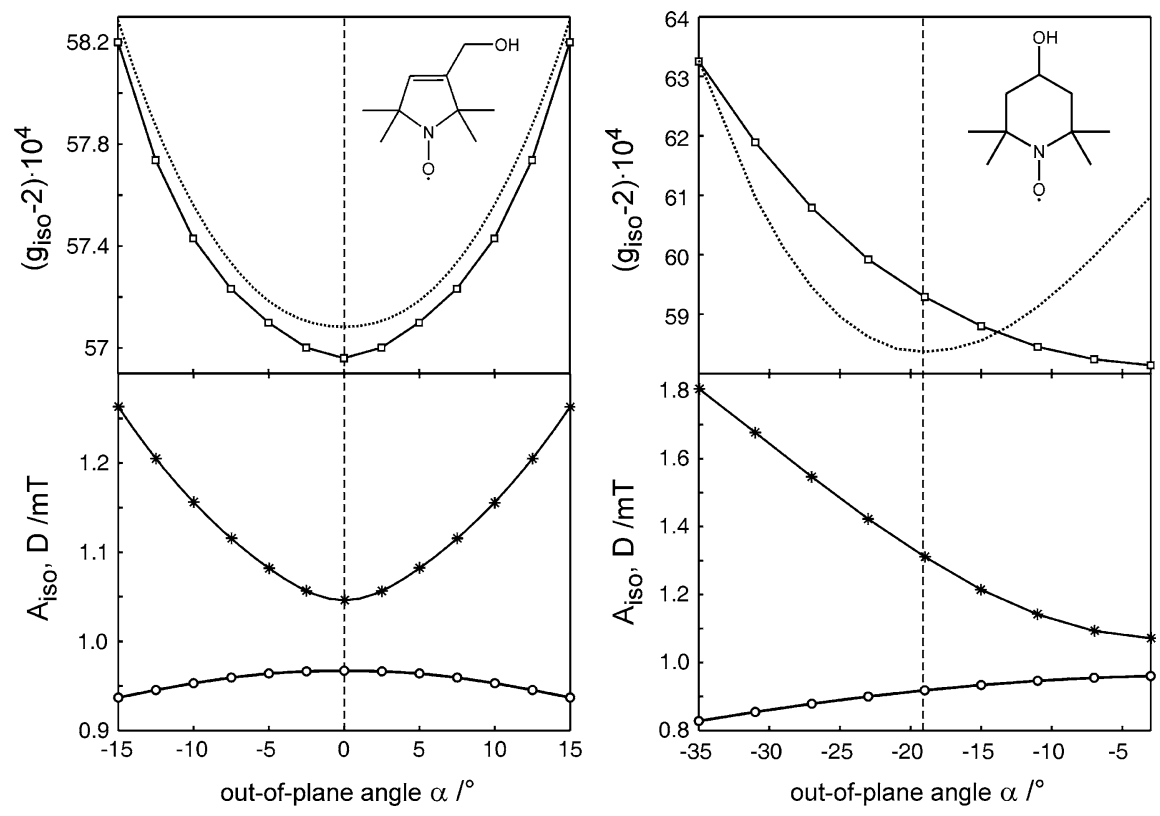

Fig. 7 The calculated $g_{\text {iso }}$ (open square), $A_{\text {iso }}$ (asterisk) and $D$ (open circle) values as a function of outof-plane angle $\alpha$ for R1 (left) and R2 (right) nitroxides. The dotted line shows the course of relaxed energy profile. The dashed line indicates the minimum energy angle. Note the different scales of the $y$ axes for R1 and R2. For details, see the text 
relaxed structures. Since increasing values of the angle $\alpha$ lead to an increasing overlap between the $p_{z}$-orbital on the $\mathrm{O}$-atom and the $s$-orbitals on the $\mathrm{N}$-atom [61], $A_{\text {iso }}$ and $g_{\text {iso }}$ exhibit an approximately parabolic dependence centered at $\alpha \approx 0^{\circ}$ in both R1 and R2. However, in relative terms, the $\alpha$-dependence of $g_{\text {iso }}-2$ (ca. $5 \%$ over $\left.10^{\circ}\right)$ is considerably weaker than that of $A_{\text {iso }}(40 \%)$.

We are omitting a detailed account of the calculated $\alpha$-dependence of the quadrupole interaction $Q$ (specifically $Q_{y y}$ ) for both $\mathrm{R} 1$ and $\mathrm{R} 2$, since this interaction parameter varies by at most $\pm 5 \%$ over an angular range of ca. $10^{\circ}$ around the respective equilibrium angles of $0^{\circ}$ and $-19^{\circ}$. This implies a relatively weak temperature dependence of $Q$ in agreement with experiment (see above). Thus, in the following, we are only considering the temperature dependence of $A_{\text {iso }}$ and $g_{\text {iso }}$.

\subsection{Temperature Dependence of $A_{\text {iso }}$ and $g_{\text {iso }}$}

Experimental results for $A_{\text {iso }}(T)$ and $g_{\text {iso }}(T)$ both for R1 and R2 (Fig. 5 and Table 1) show the opposite signs of the temperature gradients $\mathrm{d} A_{\text {iso }}(T) / \mathrm{d} T>0$ and $\mathrm{d} g_{\text {iso }}(T) /$ $\mathrm{d} T>0$ for $\mathrm{R} 1$, whereas $\mathrm{d} A_{\text {iso }}(T) / \mathrm{d} T<0$ and $\mathrm{d} g_{\text {iso }}(T) / \mathrm{d} T<0$ for R2 (although for $\mathrm{R} 2$, the temperature dependence of $g_{\text {iso }}$ is only very weak). The difference in signs of $\mathrm{d} A_{\text {iso }}(T) / \mathrm{d} T$ in $\mathrm{R} 1$ and $\mathrm{R} 2$ and in structurally similar radical types can be understood qualitatively as follows (see also Figs. 6 and 7):

R1: For symmetry reasons, this radical has a symmetrical energy profile $E(\alpha)$ around the equilibrium angle $\alpha=0^{\circ}$. The same holds true for $A_{\text {iso }}(\alpha)$ and $g_{\text {iso }}(\alpha)$. Since both these parameters show increasing values for increasing deviations from planarity, thermal vibrations around $\alpha=0^{\circ}$ will cause increasing average values of $A_{\text {iso }}$ and $g_{\text {iso }}$ towards higher temperatures. Thus, $\mathrm{d} A_{\text {iso }}(T) / \mathrm{d} T>0$ and $\mathrm{d} g_{\text {iso }}(T) /$ $\mathrm{d} T>0$.

R2: In this case, the temperature gradients of $A_{\text {iso }}(T)$ and $g_{\text {iso }}(T)$ depend on two contributions of opposite sign:

(1) The asymmetrical energy profile $E(\alpha)$ (see above) in connection with the negative slopes of both $A_{\text {iso }}(\alpha)$ and $g_{\text {iso }}(\alpha)$ around $\alpha_{\min }=-19^{\circ}$ leads to exclusively negative contributions to $\mathrm{d} A_{\text {iso }}(T) / \mathrm{d} T$ and $\mathrm{d} g_{\text {iso }}(T) / \mathrm{d} T$, since thermally activated vibrational motion towards increasing $\alpha$-values (right branch of $\left.E\left(\alpha-\alpha_{\min }\right)\right)$ is energetically favored.

(2) The increasing positive part, $\operatorname{const}\left(\alpha-\alpha_{\min }\right)^{2}$, in the $\alpha$-dependence of both $A_{\text {iso }}(\alpha)$ and $g_{\text {iso }}(\alpha)$ leads to positive contributions to both temperature gradients for the same reason as in case (1).

However, due to the fact that the slope of $A_{\text {iso }}(\alpha)$ is much larger in magnitude than that of $g_{\text {iso }}(\alpha)$, we expect the first effect to dominate only in the case of $A_{\text {iso }}(T)$ thus leading to $\mathrm{d} A_{\text {iso }}(T) / \mathrm{d} T<0$, whereas the comparitively small negative contribution to $\mathrm{d} g_{\text {iso }}(T) / \mathrm{d} T$ can easily be overcompensated by the positive contribution of the second effect.

Using the DFT results for the functions $A_{\text {iso }}(\alpha)$ and $g_{\text {iso }}(\alpha)$ as well as for the energy profiles $E(\alpha)$, we attempted a classical Boltzmann treatment similar to Lisle et al. [62] in order to get a theoretical estimate of the temperature coefficients 
$\mathrm{d} A_{\text {iso }}(T) / \mathrm{d} T$ and $\mathrm{d} g_{\text {iso }}(T) / \mathrm{d} T$ for both $\mathrm{R} 1$ and $\mathrm{R} 2$. We introduced the following simplifications:

For R1, $A_{\text {iso }}(\alpha), g_{\text {iso }}(\alpha)$ and $E(\alpha)$ were taken as pure parabolae centered on $\alpha=0^{\circ}$. For R2, the energy profile $E(\alpha)$ was split into two parabolic branches of differing slopes for $\alpha_{\min } \leq-19^{\circ}$ and $\alpha_{\min } \geq-19^{\circ}$, whereas the curves $A_{\text {iso }}(\alpha)$ and $g_{\text {iso }}(\alpha)$ were approximated by second-order polynomials. The integration over the angle $\alpha$ in the Maxwell-Boltzmann distribution function was extended from $-\infty$ to $+\infty$.

The results of these approximate calculations are presented in Table 1 for both R1 and R2. They essentially confirm the experimental results, at least in magnitude and in most of the signs. In the case of $\mathrm{R} 2, \mathrm{~d} g_{\text {iso }}(T) / \mathrm{d} T$ is extremely small in the order of $1 \times 10^{-8} \mathrm{~K}^{-1}$ and is thus theoretically not easily accessible, mainly on account of inaccuracies in the prediction of the exact energy profile by unknown solvent contributions. Such effects can enter very critically into calculations of this kind. This holds particularly for the two compensating effects (1) and (2) in the case of $g_{\text {iso }}(T)$ for R2. For a quantitative treatment, it would be necessary to include solvent effects which again require a high degree of parametrization. For a detailed discussion of the importance of solvent effects on the temperature dependence of $A_{\text {iso }}(T)$ in NO spin labels, we refer to the work of Ottaviani et al. [47].

\section{Summary and Conclusion}

A prominent field of application of nitroxide radicals is their use as spin labels attached to otherwise non-paramagnetic molecules to make them susceptible to EPR spectroscopy. Often, nitroxide spin labels are chosen for probing their microenvironment in the host matrix in terms of local dielectric and protic properties. Another important application is to probe the complex motion of both molecule and label under inner- and intermolecular constraints. As sensitive molecular probes the nitroxide-radical EPR parameters of the Zeeman, hyperfine and quadrupole interactions are well-suited, as is the line shape of the EPR spectrum. Obviously, in order to reliably use the nitroxide label as a sensitive probe for studying molecular motion phenomena of a particular guest-host system at a particular temperature, one should understand and control possible internal contributions from the probe itself that may be active in a certain temperature region overlaying other motional modes. Prototypes of EPR-detectable molecular dynamics mechanisms are, for example, overall molecular tumbling, spin-label motion around its tether by which it is bonded to the molecule, and internal motion of the nitroxide skeleton. All of these motions have specific correlation times and can principally lead to a characteristic temperature dependence of the magnetic interaction parameters. Hence, the EPR responses of the molecular complex encode this dynamic information, and sophisticated spectral analysis methods have been developed to disentangle the various modes of motion [19-23, 63].

By exploiting the enhanced resolution capabilities of $95 \mathrm{GHz}$ high-field EPR and ESEEM, in conjunction with DFT calculations on the ORCA level, we investigated the internal-motion-induced temperature dependence of the magnetic interaction parameters of the five-membered ring system of a pyrroline-type nitroxide radical 
R1, which models the head group of the commonly used MTS spin label, and of the six-membered ring system of a piperidine-type nitroxide R2 (OH-TEMPO), which models the non-natural amino-acid TOAC spin label (see Fig. 1). Ortho-terphenyl as the host matrix was chosen because its relatively high transition temperature to the glass phase allows to study the system over a wide temperature range and because ortho-terphenyl is strictly apolar and aprotic, thereby avoiding possible contributions from temperature-dependent variations of the nitroxide's matrix environment.

The temperature dependence of the dipolar (anisotropic) hyperfine coupling could be explained by the model of fast librations of the nitroxide molecule in the host matrix [18, 64]. On the other hand, the pronounced change of the isotropic hyperfine coupling with temperature is not due to an overall motion of the nitroxide molecule in the matrix, but rather originates in an intramolecular motion of the nitroxide skeleton [44]. The observed temperature coefficients of $A_{\text {iso }}$ in both R1 and $\mathrm{R} 2$ are explained by small-angle out-of-plane vibrations of the oxygen with respect to the neighboring $\mathrm{C}-\mathrm{N}-\mathrm{C}$ plane. Our DFT calculations show that the out-of-plane angle $\alpha$ of the $\mathrm{N}-\mathrm{O}$ bond is a "soft" geometrical parameter, i.e., large changes in $\alpha$ lead only to small changes in the total energy [55, 57, 61]. Nevertheless, the DFT calculations [26] reveal a strong dependence of $A_{\text {iso }}$ on this angle. An increased average squared angular deviation from the planar equilibrium geometry at the nitrogen atom with increasing temperature would explain the observed temperature behavior of $A_{\text {iso. }}$. It is important to emphasize that this distortion, however, does not influence significantly the quadrupole values.

In summary and conclusion we want to make the following statements:

Our experimental and theoretical results (see Table 1) show that the temperature dependences of $A_{\text {iso }}, g_{\text {iso }}$ and the dipolar hyperfine coupling parameter $D$ differ significantly in the two types of doublet-state nitroxide species R1 and R2.

This difference in the temperature behavior of R1 and R2 originates in their different structural properties:

In R1, which, in its equilibrium geometry, is highly symmetric and essentially planar, both $A_{\text {iso }}(\alpha)$ and $g_{\text {iso }}(\alpha)$ as well as the energy profile $E(\alpha)$ are symmetrical functions of the out-of-plane angle $\alpha$ of the $\mathrm{N}-\mathrm{O}$ bond with respect to the neighboring $\mathrm{C}-\mathrm{N}-\mathrm{C}$ plane. This structural feature, combined with thermal vibrations around the minmum energy equilibrium angle $\alpha_{\min }=0^{\circ}$, results in positive temperature coefficients of $A_{\text {iso }}$ and $g_{\text {iso. }}$.

In R2, which has a geometrical "chair" configuration, the equilibrium value of the angle $\alpha$ lies significantly below the neighboring $\mathrm{C}-\mathrm{N}-\mathrm{C}$ plane $\left(\alpha_{\min }=-19^{\circ}\right)$. This results in an asymmetrical energy profile $E(\alpha)$ around $\alpha_{\min }$; furthermore, $E(\alpha)$ is much narrower than in $\mathrm{R} 1$. Both properties lead to a significant reduction of the temperature coefficients $\mid \mathrm{d} A_{\text {iso }}(T) / \mathrm{d} T 1$ and $\operatorname{ld} g_{\text {iso }}(T) / \mathrm{d} T$. In fact, there is even a sign change in $\mathrm{d} A_{\text {iso }}(T) / \mathrm{d} T$ as a consequence of two competing factors of comparable magnitude (for details, see text above).

A classical Boltzmann treatment of thermal vibrations around $\alpha_{\min }$ for both R1 and R2 essentially confirms the experimental values for $\mathrm{d} A_{\text {iso }}(T) / \mathrm{d} T$ and $\mathrm{d} g_{\text {iso }}(T) / \mathrm{d} T$. In the case of R2, $\mathrm{d} g_{\text {iso }}(T) / \mathrm{d} T$ is extremely small (in the order of $1 \times 10^{-8} \mathrm{~K}^{-1}$ ). It is thus theoretically not easily accessible, mainly on account of inaccuracies in the prediction of the exact energy profile by unknown solvent contributions. 
In contrast to these experimental and theoretical findings concerning $A_{\text {iso }}$ and $g_{\text {iso, }}$, a potential temperature dependence of the quadrupole interaction $Q$-tensor of the ${ }^{14} \mathrm{~N}$ nucleus in the nitroxide spin label (specifically of the component $Q_{y y}$ ) remains below detectability altogether, both in R1 and R2. This fact supports our earlier statement [26] that measurements of the principal $Q$-tensor components, favorably by $95 \mathrm{GHz}$ high-field ESEEM, can well be complimentary to measurements of $A_{\text {iso }}$ and $g_{\text {iso }}$ or, preferably, of $A_{z z}$ and $g_{x x}$, in providing information on polarity effects in proteins. However, the measurement of $Q$-tensor components is not feasible in all cases. Then, this implies a restriction to the "classical" study of $A_{\text {iso }}$ versus $g_{\text {iso }}$ or $A_{z z}$ versus $g_{x x}$ "polarity plots" for obtaining information on the nitroxide's microenvironment $[5,25]$. The inherent disadvantage of this approach is that one is hampered by the non-negligible temperature dependence of these interaction parameters of the spin probe. In such a situation, comparative polarity studies with $\mathrm{R} 1$ as a spin probe should clearly be referred to identical temperatures. This requirement is less stringent when choosing $\mathrm{R} 2$ as a polarity probe on account of its significantly smaller temperature coefficients of $A_{\text {iso }}$ and $g_{\text {iso }}$ (see Fig. 5). Another implication of the temperature-dependent out-of-plane motion of the nitroxide label for EPR studies of multi-mode molecular motion under the constraints of the guestmatrix system is the suggestion to disentangle this complex motion at a fixed temperature by using a multi-frequency EPR approach $[19,63,65,66]$ rather than at a fixed EPR frequency and varying the temperature [64, 67-71].

Acknowledgments We express our gratitude to Wolfgang Lubitz for his longstanding and sustaining cooperation as a colleague and friend, and we dedicate this paper to him on the occasion of his 60th birthday. We also thank Herbert Zimmermann (Max-Planck Institute for Medical Research, Heidelberg) for having provided the protonated and deuterated forms of the nitroxide spin label R1. Financial support by the Deutsche Forschungsgemeinschaft in the frame of the Priority Program SPP 1051 ("High-field EPR in Biology, Chemistry and Physics"), the Collaborative Research Center SFB 498 ("ProteinCofactor Interactions in Biological Processes") and the Group Project MO 132/19-2 ("Protein Action Observed by Advanced EPR") is gratefully acknowledged.

Open Access This article is distributed under the terms of the Creative Commons Attribution Noncommercial License which permits any noncommercial use, distribution, and reproduction in any medium, provided the original author(s) and source are credited.

\section{References}

1. W.L. Hubbell, C. Altenbach, Curr. Opin. Struct. Biol. 4, 566-573 (1994)

2. W.L. Hubbell, A. Gross, R. Langen, M.A. Lietzow, Curr. Opin. Struct. Biol. 8, 649-656 (1998)

3. C.S. Klug, J.B. Feix, in Biological Magnetic Resonance, vol. 24B, ed. by S.R. Eaton, G.R. Eaton, L.J. Berliner (Kluwer Academic/Plenum Publishers, New York, 2005), pp. 269-308

4. G. Likhtenshtein, J. Yamauchi, S.i. Nakatsuji, A.I. Smirnov, R. Tamura, Nitroxides: Applications in Chemistry, Biomedicine, and Material Science (Wiley-VCH, Weinheim, 2008)

5. K. Möbius, A. Savitsky, High-Field EPR Spectroscopy on Proteins and their Model Systems: Characterization of Transient Paramagnetic States (Royal Society of Chemistry, London, 2009)

6. L. Columbus, W.L. Hubbell, Trends Biochem. Sci. 27, 288-295 (2002)

7. G.E. Fanucci, D.S. Cafiso, Curr. Opin. Struct. Biol. 16, 644-653 (2006)

8. H. Frauenfelder, P.W. Fenimore, R.D. Young, IUBMB Life 59, 506-512 (2007)

9. F. Gabel, D. Bicout, U. Lehnert, M. Tehei, M. Weik, G. Zaccai, Q. Rev. Biophys. 35, 327-367 (2002) 
10. G. Zaccai, Science 288, 1604-1607 (2000)

11. H. Frauenfelder, F. Parak, R.D. Young, Annu. Rev. Biophys. Biophys. Chem. 17, 451-479 (1988)

12. F.G. Parak, K. Achterhold, S. Croci, M. Schmidt, J. Biol. Phys. 33, 371-387 (2007)

13. J.C. Dyre, Rev. Mod. Phys. 78, 953-972 (2006)

14. H. Frauenfelder, G. Chen, J. Berendzen, P.W. Fenimore, H. Jansson, B.H. McMahon, I.R. Stroe, J. Swenson, R.D. Young, Proc. Natl. Acad. Sci. USA 106, 5129-5134 (2009)

15. M.M. Teeter, A. Yamano, B. Stec, U. Mohanty, Proc. Natl. Acad. Sci. USA 98, 11242-11247 (2001)

16. B.F. Rasmussen, A.M. Stock, D. Ringe, G.A. Petsko, Nature 357, 423-424 (1992)

17. D. Ringe, G.A. Petsko, Biophys. Chem. 105, 667-680 (2003)

18. S.P. Van, G.B. Birrell, O.H. Griffith, J. Magn. Reson. 15, 444-459 (1974)

19. D. Sezer, J.H. Freed, B. Roux, J. Am. Chem. Soc. 131, 2597-2605 (2009)

20. D.E. Budil, K.L. Sale, K.A. Khairy, P.G. Fajer, J. Phys. Chem. A 110, 3703-3713 (2006)

21. S.C. de Sensi, D.P. Rangel, A.H. Beth, T.P. Lybrand, E.J. Hustedt, Biophys. J. 94, 3798-3809 (2008)

22. Z.C. Liang, J.H. Freed, J. Phys. Chem. B 103, 6384-6396 (1999)

23. F. Tombolato, A. Ferrarini, J.H. Freed, J. Phys. Chem. B 110, 26248-26259 (2006)

24. M.R. Fleissner, D. Cascio, W.L. Hubbell, Protein Sci. 18, 893-908 (2009)

25. K. Möbius, A. Savitsky, C. Wegener, M. Plato, M. Fuchs, A. Schnegg, A.A. Dubinskii, Y.A. Grishin, I.A. Grigor'ev, M. Kühn, D. Duche, H. Zimmermann, H.J. Steinhoff, Magn. Reson. Chem. 43, S4S19 (2005)

26. A. Savitsky, A.A. Dubinskii, M. Plato, Y.A. Grishin, H. Zimmermann, K. Möbius, J. Phys. Chem. B 112, 9079-9090 (2008)

27. K. Möbius, A. Savitsky, A. Schnegg, M. Plato, M. Fuchs, Phys. Chem. Chem. Phys. 7, 19-42 (2005)

28. P.R. Cullis, J. Magn. Reson. 21, 397-418 (1976)

29. P.R. Cullis, J.R. Marko, Phys. Rev. B 11, 4184-4200 (1975)

30. Y.S. Lebedev, in Modern Pulsed and Continuous-Wave Electron Spin Resonance, ed. by L. Kevan, M.K. Bowman (John Wiley, New York, 1990), pp. 365-404

31. P.C. Riedi, G.M. Smith, in Electron Paramagnetic Resonance, vol. 18, ed. by B.C. Gilbert, M.J. Davies, D.M. Murphy (Royal Society of Chemistry, Cambridge, 2002), pp. 254-303

32. A. Schweiger, G. Jeschke, Principles of Pulse Electron Paramagnetic Resonance (Oxford University Press, Oxford, 2001)

33. S. Stoll, A. Schweiger, J. Magn. Reson. 178, $42-55$ (2006)

34. S. Stoll, A. Schweiger, in ESR Spectroscopy in Membrane Biophysics; Biological Magnetic Resonance, vol. 27, ed. by M.A. Hemminga, L.J. Berliner (Springer, New York, 2007), pp. 299-321

35. F. Neese, in Calculation of NMR and EPR Parameters. Theory and Applications, ed. by M. Kaupp, M. Bühl, V. Malkin (Wiley-VCH, Weinheim, 2004), pp. 541-564

36. F. Neese, EPR Newsletter 18, 10-14 (2009)

37. A.A. Shubin, G.M. Zhidomirov, J. Struct. Chem. 30, 414-417 (1989)

38. S.Y. Grebenkin, B.V. Bol'shakov, J. Phys. Chem. B 110, 8582-8586 (2006)

39. G.L. Millhauser, J.H. Freed, J. Chem. Phys. 81, 37-48 (1984)

40. M. Rohrer, P. Gast, K. Möbius, T.F. Prisner, Chem. Phys. Lett. 259, 523-530 (1996)

41. S.A. Dzuba, Y.D. Tsvetkov, A.G. Maryasov, Chem. Phys. Lett. 188, 217-222 (1992)

42. G.R. Eaton, S.S. Eaton, in Biological Magnetic Resonance, vol. 24B, ed. by S.R. Eaton, G.R. Eaton, L.J. Berliner (Kluwer Academic/Plenum Publishers, New York, 2005), pp. 3-18

43. K. Nakagawa, M.B. Candelaria, W.W.C. Chik, S.S. Eaton, G.R. Eaton, J. Magn. Reson. 98, 81-91 (1992)

44. A.T. Bullock, C.B. Howard, J. Chem. Soc., Faraday Trans. I 76, 1296-1300 (1980)

45. S. Lee, D.P. Ames, J. Chem. Phys. 81, 4206-4209 (1984)

46. B. Kirste, A. Krüger, H. Kurreck, J. Am. Chem. Soc. 104, 3850-3858 (1982)

47. M.F. Ottaviani, G. Martini, L. Nuti, Magn. Reson. Chem. 25, 897-904 (1987)

48. J.W. Turley, F.P. Boer, Acta Cryst. B 28, 1641-1644 (1972)

49. Z. Ciunik, J. Mol. Struct. 412, 27-37 (1997)

50. J. Duskova, J. Labsky, J. Hasek, I. Cisarova, Acta Cryst. E 57, O85-O86 (2001)

51. V. Zielke, H. Eickmeier, K. Hideg, H. Reuter, H.J. Steinhoff, Acta Cryst. C 64, O586-O589 (2008)

52. J. Lajzérowicz-Bonneteau, Acta Cryst. B 24, 196-199 (1968)

53. L.J. Berliner, Acta Cryst. B B 26, 1198-1202 (1970)

54. M. Cygler, Can. J. Chem. 60, 2392-2397 (1982)

55. I. Komaromi, J.M.J. Tronchet, J. Phys. Chem. 99, 10213-10220 (1995)

56. R. Improta, V. Barone, Chem. Rev. 104, 1231-1253 (2004) 
57. M. Pavone, P. Cimino, O. Crescenzi, A. Sillanpaa, V. Barone, J. Phys. Chem. B 111, 8928-8939 (2007)

58. D. Griller, J. Am. Chem. Soc. 100, 5240-5241 (1978)

59. A. Schäfer, H. Horn, R. Ahlrichs, J. Chem. Phys. 97, 2571-2577 (1992)

60. W. Kutzelnigg, U. Fleischer, M. Schindler, The IGLOO-Method: Ab Initio Calculation and Interpretation of NMR Chemical Shifts and Magnetic Susceptibilities (Springer, Heidelberg, 1990)

61. M. Plato, H.J. Steinhoff, C. Wegener, J.T. Törring, A. Savitsky, K. Möbius, Mol. Phys. 100, 37113721 (2002)

62. J.B. Lisle, L.F. Williams, D.E. Wood, J. Am. Chem. Soc. 98, 227-228 (1976)

63. P.P. Borbat, A.J. Costa-Filho, K.A. Earle, J.K. Moscicki, J.H. Freed, Science 291, 266-269 (2001)

64. S.V. Paschenko, Y.V. Toropov, S.A. Dzuba, Y.D. Tsvetkov, A.K. Vorobiev, J. Chem. Phys. 110, 8150-8154 (1999)

65. V. Bercu, M. Martinelli, C.A. Massa, L.A. Pardi, D. Leporini, J. Chem. Phys. 123, 174906 (2005)

66. V. Bercu, M. Martinelli, C.A. Massa, L.A. Pardi, D. Leporini, J. Optoelectron. Adv. Mater. 9, 17851788 (2007)

67. S.A. Dzuba, E.P. Kirilina, E.S. Salnikov, J. Chem. Phys. 125, 054502 (2006)

68. L. Andreozzi, F. Cianflone, C. Donati, D. Leporini, J. Phys. 8, 3795-3809 (1996)

69. S.A. Dzuba, E.S. Salnikov, L.V. Kulik, Appl. Magn. Reson. 30, 637-650 (2006)

70. D.A. Erilov, R. Bartucci, R. Guzzi, D. Marsh, S.A. Dzuba, L. Sportelli, Biophys. J. 87, 3873-3881 (2004)

71. D.A. Chernova, A.K. Vorobiev, J. Polym. Sci. B 47, 563-575 (2009) 